\title{
Ryan's Retinal Imaging and Diagnostics. Editor-In-Chief: Stephen J. Ryan, MD (ed): Srini Vas R. Sadda, MD 2013 (Ebook ISBN: 9780323223997, Print ISBN: 9780323262545) Saunders, an imprint of Elsevier Inc.,
}

\author{
Kaveh Abri Aghdam • Carsten Framme
}

Received: 1 November 2014 / Accepted: 5 November 2014 / Published online: 23 November 2014

(C) Springer-Verlag Berlin Heidelberg 2014

Ryan's Retinal Imaging and Diagnostics presents the first 12 chapters of Retina Fifth Edition. This book is divided into two sections: Optical Imaging Technologies and Retinal Diagnostics. Each of those sections is subdivided into six chapters. Chapter 1 and chapter 2 represent basic principles and interpretation of fluorescein and indocyanine green angiography in an appropriate structure. Clinical applications of these modalities and interpretation of angiograms are discussed in detail. Chapter 3 starts logically with the physical principles of optical coherence tomography, and the subsequent pages focus on age-related macular degeneration, but coverage of other retinal entities is less than expected. Autofluorescence imaging is the subject of chapter 4. Interpretation of fundus autofluorescence images in several retinal disorders is discussed, and accompanied with plenty of helpful images. Chapter 5 is an update on advanced imaging technologies. This chapter describes imaging of single cells in the retina, Doppler imaging, spectral imaging, photoacoustic imaging, and magnetic resonance imaging. It also introduces the emerging field of nanotechnology and nanoparticles. We would like to draw the attention of the readers to the various technologies illustrated with expressive pictures. The final chapter of section 1 details image processing, which is the field of active research in retinal imaging. Quantitative approaches to retinal image analysis are welldiscussed in this chapter. Section 2 begins with electrogenesis of the electroretinogram (ERG). Current knowledge of the cellular origins and mechanism of the various ERG component waves is reviewed. The well-constructed chapter 8 is about clinical electrophysiology. It describes concisely the method and value of electroretinogram, electro-oculogram, and visual evoked potential and their role in diagnosis, prognostic assessment, and understanding of some retinal disorders. The topic of chapter 9 is diagnostic ophthalmic ultrasound. This chapter is especially well-written, and full of practical examples and useful tables. It provides comprehensive information on ophthalmic ultrasound and its use within ophthalmology. The authors have also added schematic drawing with further details to each echogram. Describing the role of A-scan and its clinical applications in the next edition would make this chapter perfect. We recommend the reading of this chapter to all ophthalmologist colleagues. The title of chapter 10 is color vision and night vision. It reviews basic aspects of rods and cones neural pathways that process rod and cone information. There is the potential to add some clinical cases, as they have been described in less detail. Chapter 11 briefly discusses visual acuity and contrast sensitivity. The text is well-written, although it is not related to retinal imaging and could be skipped without weakening of the textbook quality. Chapter 12 , which is the final chapter of the book, reviews the role of visual fields and the principle of perimetry relevant to retinal disease. Acromatic and static short-wavelength automated perimetry as well as microperimetry are discussed here. This chapter is written skillfully and could benefit from emphasizing more on microperimetry.

Overall, this book is mainly recommended as an educational resource for retina fellowship trainees and retina specialists. It features many high-quality pictures and covers a wide range of imaging modalities and new technologies.

\footnotetext{
K. A. Aghdam • C. Framme $(\bowtie)$

Medizinische Hochschule Hannover, Carl-Neuberg-Str. 1,

30625 Hannover, Germany

e-mail: framme.carsten@mh-hannover.de
} 